\title{
Characterizing Models of Informal Physics Programs
}

\author{
Claudia Fracchiolla, ${ }^{1}$ Noah Finkelstein, ${ }^{2}$ and Kathleen Hinko ${ }^{3}$ \\ ${ }^{1}$ University College Dublin, Dublin, Ireland \\ ${ }^{2}$ University of Colorado Boulder, Boulder, CO, United States \\ ${ }^{3}$ Michigan State University, East Lansing, MI, United States
}

\begin{abstract}
There has been an increase of funding agencies' investment in informal science education in recent years, resulting in significant growth of the field. However, little research has been done in discipline-based education research to determine the impact of informal physics programs and what makes them successful. While structures exist to assess the impact of informal learning, those are not yet robust enough to rigorously assess which programs work and, more critically, why they work. In this study, we used a non-profit organization framework as a lens to evaluate the 'success' of three informal physics programs in achieving their objectives and overall vision. To determine the practices and structures that most influence the 'success' of these programs, we conducted interviews with directors and coordinators of the programs, hosted at R1 institutions and identified initial indicators that can increase chances of informal physics programs to be 'successful'.
\end{abstract}

Physicists in academia and national labs have a history of teaching physics to public audiences through informal programs. These educational programs can vary widely in terms of format, audience, content and other factors. In recent years, NSF and other funding agencies have increased their investment in informal science education partnerships and programs [1]. The result has been significant growth and variety of informal educational activities, including in physics.

As the number and diversity of informal physics programs grows, it is becoming increasingly important for administrators, faculty, and policy makers to make informed decisions on how to evaluate and support those programs. There is tension, however, since informal programs are often considered tangential to the research or teaching agenda of the organization/individual, instead of being core to the mission of the department/identity of physicist. Additionally, while the culture within physics departments can be very exclusive, in particular for women and minorities [2,3], preliminary studies indicate that participation in informal programs helps students and minorities have a stronger sense of belonging in the community $[4,5]$. We may be able to help change the culture of physics departments by integrating informal programs more into the fabric of our physics institutions.

While studies demonstrate the positive impact informal programs have on university students who facilitate the programs $[4,6]$, there is no broader understanding of the structures or practices that contribute to the 'success' of a program. Lack of a strong body of discipline-based research connected to informal physics learning prevents practitioners from being able to improve on the design of new or existing programs and administrators and faculty from understanding how best to support and provide resources to these programs.

To work toward an understanding of informal programs, we present a potential theoretical framework for understanding institutional structures and cultural practices that impact them. We draw from non-profit organizations (NPOs) effectiveness theory $[7,8]$ and adapt it to the context of informal physics programs. This framework is expansive. In this paper, we narrow the scope to focus on the impact of funding on all aspects of program design and implementation, on a sam- ple data set and report on the efficacy of the framework. This work is a pilot study for a national scale project investigating informal physics programs.

\section{FRAMEWORK}

We take a socio-cultural perspective of learning and being in the world when considering how informal programs operate within institutions. This lens allows for a dynamic understanding of how entities and the people in them are affected. In this study, we present a NPO framework for organizational effectiveness [8] to be used to describe informal physics programs. This theory, from business literature, attempts to predict and explain how organizations and people within them will behave in varying structures, cultures, and circumstances [7]. For these reasons, we postulate that the NPO framework could be a valuable tool to understand the diversity and complexity of informal physics programs.

The NPO framework identifies relevant dimensions to determine the 'success' of an organization. In this study, success is connected to the longevity and sustainability of the program, achievements of the program aligned with its goals, impact that the program has beyond traditional measures, and the programs' own perception of success. Table I contains the descriptions of the NPO dimensions which we have contextualized to informal physics programs. For instance, board was changed to stakeholder to be flexible for different types of program structures. To test the efficacy of the framework, we use the dimensions to identify factors that contribute or hinder the function of three informal physics programs.

\section{RESEARCH DESIGN}

This work uses a multi-case study approach. Three programs are studied in depth to understand how institutional structures and cultural practices of institutions impact the success of the programs, through the lens of the NPO effectiveness framework. The programs were selected because they 
TABLE I. Elements of NPO framework contextualized to informal physics programs

\begin{tabular}{ll}
\hline Dimensions & Description \\
\hline Stakeholders & $\begin{array}{l}\text { Related to structure, homogeneity, and experience of the stakeholders (board). Indicators are account- } \\
\text { (SD) }\end{array}$ \\
\hline $\begin{array}{l}\text { Managility, liability and responsibility } \\
\text { (MD) }\end{array}$ & $\begin{array}{l}\text { Related to mission success and achievement of goals; organizational flexibility to adapt to changes, the } \\
\text { strategies and actions to achieve goals, professionalism, and organizational learning }\end{array}$ \\
\hline $\begin{array}{l}\text { Resource } \\
\text { (RD) }\end{array}$ & $\begin{array}{l}\text { Related to tangible resources (funding, access to spaces), intangible resources (volunteers, administrative } \\
\text { support), capacity to maximize the use of resources, and the management of human resources }\end{array}$ \\
\hline Finance (FD) & $\begin{array}{l}\text { Related to financial performance (budget, income, and expenses) as well as finance health (mechanisms } \\
\text { to evaluate the use of resources) }\end{array}$ \\
\hline $\begin{array}{l}\text { Environment } \\
\text { (ED) }\end{array}$ & $\begin{array}{l}\text { Related to the technical environment, such as training and professional development, as well as social } \\
\text { environment (cultural practices, building community) }\end{array}$ \\
\hline Program (PD) & $\begin{array}{l}\text { Related to the success of programs/projects that have been delivered, the service quality and stakehold- } \\
\text { ers' satisfaction with program), and measurable outcomes ( activities, workshops, student learning) }\end{array}$ \\
\hline
\end{tabular}

are: i) successful under our definition; ii) accessible for the researchers; iii) well developed (two of the programs have been running for over 5 years); and iv) housed at similar institutions (R1), but represent a variety of goals and formats.

To gain deep insight into the history and development of each program, we designed a semi-structured interview protocol, using the NPO framework dimensions as guidelines. For example, questions from the protocol regarding the finance dimension include: How is the program funded? Has that changed? Why? How has that impacted the program? Interviews were conducted with directors and advisors of the programs and lasted on average 1.5-2 hours.

Interviews were analyzed using content analysis to identify emergent themes; themes were used as seed to create concept maps, which show how themes connect to different elements of the programs. Next, the concept maps were coded with the adapted NPO framework. The use of a flexible approach, such as concept maps for analysis, allows to graphically organize the interconnectivity of the elements that have an effect on the programs' success. Furthermore, the use of maps facilitates the framing of experiences from a participant-centric perspective and visually orients researchers to ground experience in theory [9]. Reliability was achieved by having the researchers independently revise and compare the maps and coding, then discuss and resolve differences.

\section{FINDINGS}

From the concept map analysis several key themes emerged, including institutional connections, personnel, resources, and funding. In this study we focus on the funding element because: i) it was one of the most important factors for every programs' success, ii) it connected to different $N P O$ dimensions, and iii) it was imperative for the sustainability of the program and the support of physics students. As one subject says: "If you remove the [center's] funding [the outreach program] wouldn't run." Here we describe each program and indicate in parentheses the NPO dimensions relevant to elements of the its trajectory.

Program 1 is a traveling physics and science demonstration program where undergraduate students design and lead hands-on guided exploration activities for K-8 children. The interviewee was the founder and director, who has transitioned into more of a management role.

The source of funding for Program 1 has drastically changed on three occasions, which has affected where the program is housed within the institution, as well as the focus and structure of the program (FD). With each change of funding, the program had to evolve to meet the requirements of the funding group, in terms of goals and content. The imposed priorities required restructuration and evaluation, from which new activities and collaborations were developed. However, these changes do not seem to have hurt the growth of the program. On the contrary, the program has significantly grown in terms of the number of children reached and partnerships with teachers and schools, as well as the activities they engage in. According to the program director, the funding changes have helped them create a more well rounded program:

\footnotetext{
"The main goals have been pretty consistent. There are three elements of our priorities and we represent it as a pie. The size of the priorities has changed but the priorities themselves haven't."
}

Figure 1 shows the coded map of Program 1 using the NPO framework. The emergent elements from the history of the program and connections between them are represented in the three main columns. The column on the middle represents the different funding sources the program have had access to. The main column to the right indicates the things the program achieved with the corresponding funding source. Finally, main column to the left indicates how the program aligned their goals to those of the funding sources. The color labels indicate the NPO codes. The colors on the labels indicate the NPO dimension (See legend).The names in the labels indicate which category within the dimension it was coded with 
FIG. 1. Concept map of funding theme for Program 1

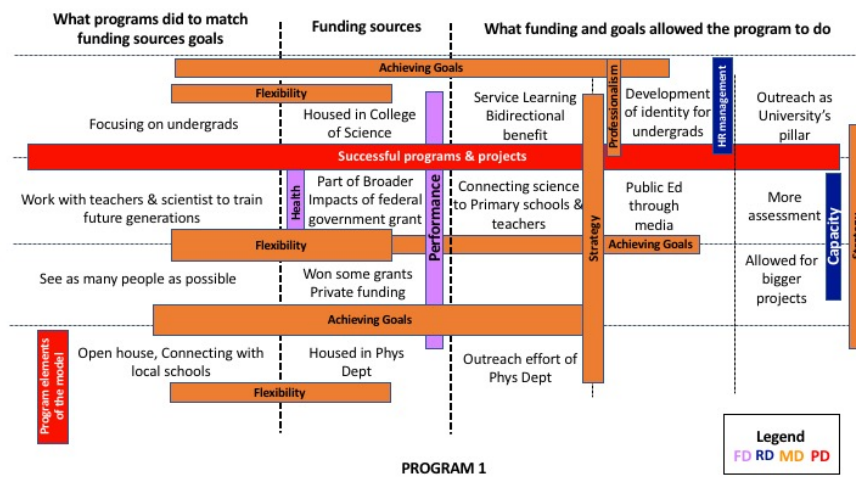

and the length and with of the labels connect the elements of the program coded with the shown label. Through the use of the framework on the maps we can highlight the elements of funding that have had bigger impact on the program's success. For example, the program has not had a constant source of funding throughout the years (FD); this directly connects to the organizational flexibility of the program (MD), which has had to redirect their focus to work with different sources of funding. To meet the goals of the funding agencies, they have had to adapt their strategies to achieve those goals (MD), such as developing a Service Learning component to their program (MD, PD). Also in response to funding, they have needed to improve the professional development of their most valuable resource, the undergraduate students who facilitate the program and, in particular, to provide them with opportunities to have positive experiences and foster their identity (ED, RD, PD). This change led to improved job satisfaction for the facilitators and new sources of funding with partners that supported those initiatives (PD, MD). Finally, the board comprised an in-house team to maintain some consistency through the funding transitions (SD).

Program 2 is an afterschool physics program, where mostly graduate physics students meet weekly with K-12 youth to engage in hands-on activities. The interviewee was a main driver of the current model of the program and now closely advises program staff rather than run daily operations.

Unlike Program 1, the funding for this program comes from one source, a national physics center, and has stayed consistent for over a decade (FD). The center provides stable funding for a full-time staff position, supplies for the activities, and resources such as the machine shop and IT support (RD). The interviewee describes the program as "officially sanctioned by [the center] and built into the [center's] funding." Because of the nature of the funding source, a representative of the center representative acts as an advisor of the program (SD). Importantly, the main goal of their funders has also stayed constant - it is simply a mission to engage the center in public outreach (MD). This open-endedness has allowed program personnel to define for themselves more spe- cific goals for the program, such as expanding the research element or adding STEAM programs (PD). They have supplemented the main funding source by strategically targeting additional small funding sources, which have been awarded at different stages, to run these specific projects (FD). The result of these small awards has been an increase on the research element of the program and the number of people doing the research (PD). Thus, the program has had moderate growth over the years, gaining a staff director, coordinator, and interns, as well as serving increasing numbers of youth (RD).

An important characteristic of Program 2 that has a big impact on funding is the emphasis on working with community partners (FD), who absorb some of the cost that would be included in running the program, such as the spaces where the program is run, as well as the support of teachers (PD). This reduced cost allows the program to run weekly and also to run at more than one site per semester. In this way, partnership helps the program achieve the goals of both the funders and community partners (MD): the main funder's goal of having an outreach effort is fulfilled; the community partners achieve their goal of providing opportunities for children to engage in STEM activities; and the informal program through constant and long exposure is achieving its goal of building scientific identity for the children and the facilitators, as well as changing the culture of the physics research center and their connection with the community (MD).

As with Program 1, Program 2 is embedded in a R1 institution, which provides them access to tangible and intangible resources (RD). However, unlike Program 1, the access to these resources, as well as the spaces provided by the partnership are key to their funding and success.

Program 3 is a city-wide campaign to promote conversations about physics on the streets and make physics part of daily culture. The campaign ran twice, a year apart, and was considerably bigger the second time. The interviewee was the co-creator of the program. He worked on every aspect of the program: conceptualizing the idea, identifying and obtaining funding, finding partners, and shepherding the program from start until completion.

The nature of the funding of Program 3 differs from that of Programs $1 \& 2$, since it was a campaign that happened during a set period of time. It only required funding for the implementation of the idea, instead of continuous funding (FD). However, because the campaign had different elements (RD), it required diverse and simultaneous sources of funding (FD). For example, the development and conceptualization of the idea was possible through seed funding, which gave the creator the opportunity to take the idea to proof of concept, which was used to win a grant for a main source of funding. The grant supported the core elements of the campaign (MD). Smaller sources of funding facilitated the reach and success the campaign had (FD, RD). Like Program 1, Program 3 had to satisfy different funders' goals, but this was accomplished without shifting the focus of the program's mission (MD). For example, the main funder's goal was to reach a large amount of people; however, this objective did not interfere but con- 
tributed to the mission of engaging people with physics and making physics part of culture.

Like Program 2, Program 3 worked under partnership principles (MD, FD). Funders used the partnership for individual growth (PD). For example, one of the funders was a company that designed some of the products for the campaign. The company provided discounts for the work in exchange to use the products created as an advertisement tool for themselves. Another aspect of the partnership model was creating a social environment that built community beyond the collaboration (ED). For example, a transit company agreed to discount advertising spaces, because it believed that the campaign would help build identity and community within their clients.

Finally, Program 3 was housed at an R1 institution, which provided institutional support but comes with constraints, as with the other programs (FD, RD). However, like Program 1 , because there were several sources of funding, the main team that managed the program was also responsible for the program's structure and cultural practices (SD).

\section{DISCUSSION}

From the NPO analysis, we identified a key characteristic of the success for the three programs: they are not limited by their funding sources because they adapt, evolve or identify different sources of funding to complete their vision, expand their goals, test different projects, or develop new elements of the program. The programs' position within the institutions in which they are hosted is different, as are the institutional structures; therefore, the impact of funding on each program is also different. Some characteristics of institutional structures are directly linked to the funding and impact aspects, such as growth, content, participants, and goals of the program. We have identified similarities and differences across the programs that have significantly affected their success:

Finance Dimensions: The programs used different sources of funding, which filled different purposes within the structure of the programs. They all faced constraints regarding the use of funding from external (federal) organizations.

Program and Management Dimensions: The programs have different goals - aligned with the sources of funding and strategies to deliver them without steering much from the programs' mission. Program l's strategy is linked to their adaptability; shifting their focus and evaluating strategies to best achieve the new goals, which reflects on the management dimensions. Program 1 also used their organizational learning to their advantage, by identifying a funder who would invest on the development of the facilitators (university students). Programs 2 and 3 connected strategy to their partnerships model, going beyond achieving goals, to benefit the partners' personal interests and satisfy stakeholders.

Resources Dimensions: The programs are housed at R1 institutions and have access to similar tangible (funding, spaces) and intangible (volunteers, administrative support) resources, which are key for sustainability. Even though the director of Program 1 did not talk about those resources directly, he described the intentionality with which they maximized the use of their limited resources. For example, Program 1 found funding by developing strategies to create job satisfaction for their volunteers. By making undergraduate students happy, the program is helping attract and keep more students, which in turn makes the institution consider the program as a "personal" investment.

In conclusion, we have shown that the NPO framework is a viable tool to understand how institutional structures and cultural practices shape informal physics program's success. Going forward, we will look at other key features like the culture of the volunteers and program practices. We intend to apply the modified NPO framework to a large data set of informal programs to illuminate our understanding of the state of informal physics in the U.S. and inform university support for and design of informal physics programming.

Another contribution of this work is its implications for practice. Preliminary findings indicate that programs have to be able to shift focus and adapt to changes to have access to broader potential sources of funding. Programs also need to identify sources of funding and partners, in which the mission of the program and the goals of the funder are not in complete misalignment. Identifying partnerships means that the programs can deliver what they are asked, guarantee the core mission of the program, and satisfy stakeholders and personnel, which is very important for the sustainability of the program. By continuing to use the NPO framework to understand a wider scope of informal programs, we believe we will be able to draw out best practices and recommendations for physics administrators, faculty and staff in their decisions about resources, program design, and assessment.

This work is supported by the JILA NSF Physics Frontier Center (\#1125844) and NSF AISL award (\#1423496).
[1] National Research Council. (2015). Identifying and supporting productive STEM programs in out-of-school settings. National Academies Press.

[2] Hyater-Adams, S., et al. (2018). Phys Rev PER, 14(1), 010132.

[3] Potvin, et al. (2017). Phys Rev PER, 13(2), 020142.

[4] Fracchiolla, C., et all. (2016) PERC Proceedings, p124-127. Sacramento, CA. July 20-21, 2016.

[5] Dabney, K. P., et al. (2012). Int. J. Sci. Ed., Part B, 2(1), 63-79.

[6] Hinko, K. A., et al. (2016). Phys Rev PER, 12(1), 010111.
[7] Shafritz, et all. (2015). Classics of organization theory. Cengage Learning.

[8] Taysir, E. A., \& Taysir, N. K. (2012). Journal of Transnational Management, 17(3), 220-235.

[9] Wheeldon, J., \& Faubert, J. (2009). International Journal of Qualitative Methods, 8(3), 68-83.

[10] Kuh, G. D. (2003). Mag. of Higher Learning, 35(2), 24-32. 